\title{
Oscilador Harmônico Quântico 1D Confinado e sua Energia Variacional obtida com Auxílio da Supersimetria
}

E. DRIGO FILHO ${ }^{1}$, Departamento de Física, IBILCE, UNESP, 15054-000 São José do Rio Preto, SP, Brasil.

R.M. RICOTTA2, Departamento de Ensino Geral, FATEC/SP - CEETEPS UNESP, 01124-060 São Paulo, SP, Brasil.

Resumo. O estado fundamental do oscilador harmônico quântico unidimensional confinado é determinado através do Método Variacional aliado ao formalismo da Mecânica Quântica Supersimétrica. São apresentados resultados numéricos para vários valores do raio de confinamento. Observa-se que o autovalor de energia para o problema não confinado é recuperado quando este raio se torna muito grande.

\section{Introdução}

Os recentes avanços tecnológicos relacionados à nanotecnologia, como nanotubos, nanofios e pontos quânticos, têm renovado o interesse em sistemas quânticos confinados em regiões espacialmente limitadas. Nesta direção, como exemplo de aplicação tecnológica, pode-se citar o uso de pontos quânticos em semicondutores, [15].

Paralelamente ao desenvolvimento e aplicação tecnológica, esforço tem sido desprendido no estudo teórico de sistemas confinados; um exemplo é o tratamento do átomo de hidrogênio, [22], em situações onde há confinamento. Neste sentido, diferentes técnicas matemáticas, como a expansão 1/N, [18], o método WKB, [19], e o método perturbativo, [1], têm sido empregadas objetivando a determinação dos níveis de energia de tais sistemas. Particularmente, o método variacional tem sido bastante lembrado e aplicado neste tipo de problema (vide referências [14], [22] e [23] e trabalhos lá citados). A abordagem tradicional deste método consiste em encontrar uma função de onda para ser usada como teste na obtenção do valor esperado da energia. Uma inovação neste tratamento foi sugerida baseada no uso da supersimetria em Mecânica Quântica.

O formalismo resultante da Mecânica Quântica Supersimétrica, MQS, [4, 5, $12,13]$, tem sido usado com muito sucesso na análise de problemas quânticos; vale destacar o tratamento de potenciais exatamente, [2, 20, 21], e parcialmente solúveis, $[6,7,16]$. Usando este formalismo é possível encontrar um potencial efetivo e,

\footnotetext{
${ }^{1}$ elso@df.ibilce.unesp.br; apoio financeiro parcial do CNPq.

${ }^{2}$ regina@fatecsp.br
} 
a partir dele, a função de onda teste a ser usada no método variacional. O elo entre potencial e função de onda é feito através do chamado superpotencial. Esta linha de raciocínio foi primeiro usada no estudo de sistemas não confinados, $[3,8$, 9], e, mais recentemente, para os sistemas confinados, [10, 11]. Entretanto, até o momento apenas sistemas confinados tridimensionais têm sido tratados usando o método variacional aliado à MQS.

Neste trabalho são determinados variacionalmente os autovalores de energia do estado fundamental para o oscilador harmônico unidimensional para vários valores do raio de confinamento $R$. Uma modificação na forma do superpotencial utilizado é sugerida em relação ao caso tridimensional. Também é mostrado que o resultado variacional coincide com o valor encontrado analiticamente para o problema sem confinamento quando $R$ se torna muito grande.

\section{MQS}

No formalismo da MQS, $[4,12,13,5]$, com $N=2$ têm-se dois operadores $Q$ e $Q^{+}$ que satisfazem as seguintes relações de anti-comutação:

$$
\{Q, Q\}=\left\{Q^{+}, Q^{+}\right\}=0, \quad\left\{Q, Q^{+}\right\}=H_{s s},
$$

onde $H_{s s}$ é o Hamiltoniano supersimétrico. A realização usual destes operadores é feita através de matrizes $2 \times 2$, a saber:

$$
Q=\left(\begin{array}{cc}
0 & 0 \\
A^{-} & 0
\end{array}\right), \quad Q^{+}=\left(\begin{array}{cc}
0 & A^{+} \\
0 & 0
\end{array}\right) .
$$

Os operadores $A^{ \pm}$são chamados operadores bosônicos. Considerando a dependência em apenas uma variável $(x)$, estes operadores podem ser escritos como:

$$
A^{ \pm}=\frac{1}{\sqrt{2}}\left(\mp \frac{d}{d x}+W(x)\right),
$$

onde $W(x)$ é chamado de superpotencial.

Com a estrutura indicada acima, o Hamiltoniano supersimétrico é escrito como uma matriz diagonal $2 \times 2$, ou seja:

$$
H_{S S}=\left(\begin{array}{cc}
A^{+} A^{-} & 0 \\
0 & A^{-} A^{+}
\end{array}\right)=\left(\begin{array}{cc}
H^{+} & 0 \\
0 & H^{-}
\end{array}\right) .
$$

Os operadores $H^{ \pm}$são conhecidos como companheiros supersimétricos e possuem a seguinte forma:

$$
\begin{aligned}
H^{ \pm} & =-\frac{h^{2}}{2 m} \frac{d^{2}}{d x^{2}}+V_{ \pm}(x) \\
& =-\frac{h^{2}}{2 m} \frac{d^{2}}{d x^{2}}+\frac{1}{2}\left(W^{2}(x) \mp W^{\prime}(x)\right) .
\end{aligned}
$$

onde $W^{\prime}=\frac{d W}{d x}$. 
A estrutura algébrica da MQS permite várias aplicações no estudo de sistemas quânticos, em particular, ela pode ser usada como base para um método de solução da equação de Schrödinger. Este método inicia-se com a fatoração de um dado Hamiltoniano em termos dos operadores bosônicos (2.3). Desta forma, partindo-se de um Hamiltoniano original $H_{0}$,

$$
H_{0}=-\frac{h^{2}}{2 m} \frac{d^{2}}{d x^{2}}+V_{0}(x),
$$

deve-se procurar por um superpotencial que permita escrever este operador como $H^{+}=A^{+} A^{-}$. Isto pode ser conseguido resolvendo a seguinte equação de Ricatti para o superpotencial, ou seja, obtendo a solução para $W(x)$ na equação:

$$
V_{0}(x)=\frac{1}{2}\left(W(x)^{2}-\frac{d W(x)}{d x}\right)+E_{0} .
$$

Esta relação segue diretamente da definição dos Hamiltonianos companheiros supersimétricos em (2.5). O termo constante $E_{0}$ é o autovalor de energia do estado fundamental, sua adição garante a construção do Hamiltoniano como um produto dos dois operadores bosônicos. Por conveniência, optou-se por usar $m=h=1$. Essa escolha particular de unidades simplifica a notação sem afetar a generalidade dos resultados apresentados.

Um aspecto fundamental do presente tratamento é que a super-álgebra garante que:

$$
A^{-} \Psi_{0}(x)=0,
$$

ou seja, usando a definição (2.3):

$$
\Psi_{0}(x)=N \exp \left(-\int_{0}^{x} W(y) d y\right) .
$$

onde $N$ é a constante de normalização.

O processo descrito acima para a solução do estado fundamental da equação de Schrödinger pode ser estendido para estados excitados através da hierarquia de Hamiltonianos, $[2,4,5,12,13,20,21]$. Entretanto, para os propósitos deste trabalho basta a descrição do tratamento para o estado fundamental.

Nem todos os potenciais $\left(V_{0}(x)\right)$ de interesse permitem obter a solução analítica da equação de Ricatti (2.7). Quando esta solução não é possível, a equação (2.7) permite uma busca sistemática por soluções aproximadas para o superpotencial. Esta busca é feita determinando-se potenciais efetivos $\left(V_{e f}(x)\right)$ similares ao potencial original. Uma vez que o superpotencial aproximado seja escolhido, a relação (2.9) fornece automaticamente uma função de onda aproximada para o problema. Esta função pode ser usada no Método Variacional.

\section{Método Variacional}

O Método Variacional tem sido amplamente usado como método aproximativo para determinação de autovalores de energia, principalmente para o estado fundamental. O formalismo e a fundamentação matemática para sua aplicação podem ser 
encontrados em diversos livros textos de Mecânica Quântica (vide, por exemplo, a referência [17]). Em essência, uma função de onda teste deve ser usada para determinar um valor esperado da energia; a minimização da energia em termos dos parâmetros da função teste fornece um limite superior para o autovalor da energia procurado. A determinação da função de onda teste é o ponto chave desta abordagem; quanto melhor a qualidade desta função melhor serão os resultados obtidos.

Em termos matemáticos, o valor esperado da energia é dado por:

$$
E_{\mu}=\frac{\int \Psi_{\mu}^{*} H_{0} \Psi_{\mu} d x}{\int\left|\Psi_{\mu}\right|^{2} d x}
$$

onde $H_{0}$ é o Hamiltoniano original e $\Psi_{\mu}(x)$ é a função de onda teste que depende do conjunto de parâmetros $\{\mu\}$. O valor esperado da energia $E_{\mu}$ deve ser minimizado em termos destes parâmetros. A integral no denominador corresponde à normalização de $\Psi_{\mu}(x)$. Os extremos de integração dependem do problema sob análise no caso de interesse, ou seja, no confinamento em uma dimensão esses extremos vão de $-R$ até $+R$, onde $R$ é o raio do confinamento.

A contribuição da super-álgebra para o Método Variacional está na determinação da função de onda teste, como descrito no último parágrafo da seção anterior. Assim, através da escolha do superpotencial e do cálculo da função de onda, equação (2.9), o potencial efetivo, semelhante ao potencial original, é obtido, sendo dado por

$$
V_{e f}(x)=\frac{1}{2}\left(\bar{W}^{2}-\bar{W}^{\prime}\right)+E(\bar{\mu})
$$

onde $\bar{W}=W(\bar{\mu})$ é o superpotencial para $\{\mu=\bar{\mu}\}$, o conjunto de parâmetros que minimiza (3.1).

\section{Oscilador Harmônico Unidimensional Confinado}

O sistema específico a ser estudado é o oscilador harmônico unidimensional confinado na região $-R<x<R$. A função potencial para este sistema é escrita como:

$$
\begin{array}{rlrl}
V(x) & =\frac{1}{2} x^{2}, & -R<x<R \\
& =+\infty, \quad x= \pm R .
\end{array}
$$

Assim, o Hamiltoniano para o oscilador harmônico dentro da região de confinamento, em unidades atômicas, fica sendo:

$$
H=-\frac{1}{2} \frac{d^{2}}{d x^{2}}+\frac{x^{2}}{2} .
$$

O confinamento é produzido por uma barreira infinita na posição $x= \pm R$. O limite para que o sistema possa ser considerado não confinado ocorre quando o raio $\mathrm{R}$ assume valores grandes. O sistema não confinado permite solução para todos os níveis de energia; particularmente o estado fundamental possui autovalor de energia 
igual a $\frac{1}{2}$, nas unidades adotadas [17]. O superpotencial sugerido para este problema é

$$
W(x)=-\frac{\mu_{1}}{x-R}-\frac{\mu_{2}}{x+R}+\mu_{3} x,
$$

que depende de três parâmetros $\left(\mu_{1}, \mu_{2}, \mu_{3}\right)$ que serão usados como parâmetros variacionais. Os primeiros dois termos do superpotencial garantem que o potencial, obtido pela equação de Ricatti (2.7), vá para infinito quando $x \rightarrow R$. É importante notar que o potencial nesta abordagem pode ser escrito a menos de uma constante aditiva que, no caso de solução exata, corresponderia ao próprio autovalor de energia como indicado em (2.7). O último termo do superpotencial corresponde ao termo usual quando não há confinamento, [2].

Explicitamente, o potencial efetivo, dado pela equação (3.2), tem a seguinte forma

$$
\begin{aligned}
V_{e f}(x)= & \frac{\bar{\mu}_{3}^{2}}{2} x^{2}+\frac{\bar{\mu}_{1}\left(\bar{\mu}_{1}-1\right)}{2(x-R)^{2}}+\frac{\bar{\mu}_{2}\left(\bar{\mu}_{2}-1\right)}{2(x+R)^{2}}-\frac{\bar{\mu}_{1} \bar{\mu}_{3} x}{x-R}-\frac{\bar{\mu}_{2} \bar{\mu}_{3} x}{(x+R)} \\
& +\frac{\bar{\mu}_{1} \bar{\mu}_{2}}{x^{2}-R^{2}}-\frac{\bar{\mu}_{3}}{2}+E(\bar{\mu}),
\end{aligned}
$$

que diverge quando $x= \pm R$, como esperado no sistema confinado. Note-se que o potencial efetivo é calculado para valores do conjunto de parâmetros $\{\bar{\mu}\}$ que minimizam a energia.

A função dada em (4.3) difere dos superpotenciais usados para confinamento em três dimensões, [10,11], pela inclusão de um termo a mais de barreira de potencial para quando $x \rightarrow-R$. Esse termo é desnecessário no estudo de equações radiais em três dimensões, uma vez que a variável radial jamais poderá assumir valores negativos.

Usando a relação entre superpotencial e função de onda (2.9) obtém-se a função teste:

$$
\Psi_{\mu}(x)=(x-R)^{\mu_{1}}(x+R)^{\mu_{2}} e^{-\mu_{3} x^{2} / 2} .
$$

Essa função permite determinar variacionalmente autovalores de energia para o sistema estudado para diferentes valores do raio de confinamento.

A energia é então obtida pela minimização do valor esperado da energia com relação a $\mu$. Assim, a equação a ser minimizada é dada por

$$
E_{\mu}=\frac{\int_{-R}^{R} \Psi_{\mu}(y)\left[-\frac{1}{2} \frac{d^{2}}{d x^{2}}+\frac{x^{2}}{2}\right] \Psi_{\mu}(y) d y}{\int_{-R}^{R} \Psi_{\mu}(y)^{2} d y} .
$$

Os resultados obtidos, usando na equação (4.6), o Hamiltoniano (4.2) e a função (4.5) estão mostrados na Tabela 1. Observa-se que quando o valor do raio $R$ está próximo de 6,5 o sistema já pode ser considerado não confinado no que se refere ao estado fundamental. 


\begin{tabular}{|c|c|}
\hline $\begin{array}{c}\text { Raio de Confinamento } \\
\mathrm{R}\end{array}$ & $\begin{array}{c}\text { Energia Variacional } \\
E_{\text {variacional }}\end{array}$ \\
\hline 1.0 & 1.29847 \\
\hline 2.0 & 0.537913 \\
\hline 3.0 & 0.500618 \\
\hline 4.0 & 0.500028 \\
\hline 4.5 & 0.500009 \\
\hline 5.0 & 0.500004 \\
\hline 5.5 & 0.500002 \\
\hline 6.0 & 0.500001 \\
\hline 6.31896 & 0.500000 \\
\hline
\end{tabular}

Tabela 1: Resultados para os autovalores de energia (em Rydbergs) para o oscilador harmônico unidimensional confinado com diferentes valores do raio de confinamento $R$. Os autovalores foram obtidos usando Método Variacional com funções de ondas testes fornecidas pelo superpotencial.

\section{Conclusão}

O tratamento apresentado neste trabalho consiste no uso do Método Variacional aliado ao formalismo da MQS. Observa-se que os resultados para sistemas confinados tridimensionais, $[10,11]$, podem ser adaptados para tratamento de sistemas unidimensionais.

Os resultados numéricos obtidos são consistentes com o esperado. O efeito do confinamento é significativamente mais pronunciado para valores pequenos do raio de confinamento. A medida que esse raio aumenta o sistema se aproxima do caso não confinado. Uma vez que o valor de $\mathrm{R}$ se torne suficientemente grande, o autovalor de energia coincide com o caso não confinado, ou seja, $\mathrm{E}=\frac{1}{2}$ para o estado fundamental.

A metodologia usada no presente trabalho, apesar de simples, se mostra bastante útil, tendo sido aplicada em vários contextos com sucesso. Os resultados obtidos indicam que problemas envolvendo sistemas unidimensionais confinados também podem ser proveitosamente estudados através da abordagem proposta.

\section{Agradecimentos}

Os autores agradecem à Prof. Dra. Socorro Rangel pela discussão dos tópicos apresentados neste trabalho.

Abstract. The lowest energy level of the one-dimensional confined quantum har-
monic oscillator is determined through the Variational Method associated to the
formalism of Supersymmetric Quantum Mechanics. Numerical results for different
values of the radius of confinement are presented. It is observed that the energy
eigenvalue of the non-confining problem is recovered when this radius becomes large. 


\section{References}

[1] V.C. Aguilera-Navarro, J.F. Gomes, A.H. Zimerman e E. Ley Koo, On the radius of convergence of Rayleigh-Schrödinger perturbative solutions for quantum oscillators in circular and spherical boxes, J. Math. Phys.: Math. Gen. A, 16 (1983), 2943-2952.

[2] G.R.P. Borges e E. Drigo Filho, Supersimetria em Mecânica Quântica II: Oscilador Harmonico e Potencial Coulombiano, Rev. Bras. Ens. Fis., 21 (1999), 233-237.

[3] G.R.P. Borges e E. Drigo Filho, Supersymmetry, Variational Method and the Lennard-Jones (12,6) Potential, Int. J. Mod. Phys. A, 16 (2001), 4401-4407.

[4] F. Cooper, A. Kare e U.P. Sukatme, "Supersymmetric Quantum Mechanics", World Scientific, Singapure, 2001.

[5] E. Drigo Filho, Supersimetria em Mecânica Quântica, Rev. Bras. Ens. Fis., 19 (1997), 152-158.

[6] E. Drigo Filho e R.M. Ricotta, Partial Algebraization of the Non-Polynomial Potential, Mod. Phys. Lett. A, 6 (1991), 2137-2142.

[7] E. Drigo Filho e R.M. Ricotta, Supersymmetric Quantum Mechanics and Partially Solvable Potential, Phys. At. Nucl., 61 (1998), 1722-1725.

[8] E. Drigo Filho e R.M. Ricotta, Supersymmetry, Variational Method and Hulthen Potential, Mod. Phys. Lett. A, 10 (1995), 1613-1618.

[9] E. Drigo Filho e R.M. Ricotta, Morse Potential Energy Spectra through the Variational Method and Supersymmetry, Phys. Lett. A, 269 (2000), 269-276.

[10] E. Drigo Filho e R.M. Ricotta, Supersymmetric Variational Energies of 3d Confined Potentials, Phys. Lett. A, 320 (2003), 95-102.

[11] E. Drigo Filho e R.M. Ricotta, Supersymmetric Variational Energies for the Confined Coulomb System, Phys. Lett. A, 299 (2002), 137-143.

[12] L.E. Gendenshtein e I.V. Kive, Supersymmetry in Quantum Mechanics, Sov. Phys. Usp., 28 (1985), 553-590.

[13] R.W. Haymaker e A.R.P. Rau, Supersymmetry in Quantum-Mechanics, Am. J. Phys., 54 (1986), 928-936.

[14] J.L. Marin e S.A. Cruz, Enclosed Quantum-Systems - Use of the Direct Variational Method, J. Phys. B: At. Mol. Opt. Phys., 24 (1991), 2899-2907.

[15] S.Y. Ren, Quantum confinement in semiconductor Ge quantum dots, Solid State Comm., 102 (1997), 479-484. 
[16] P. Roy e R. Roychoudhury, New Exact-Solutions of the Nonpolynomial Oscillator $\mathrm{V}(\mathrm{x})=\mathrm{x} 2+$ Lambda-x $2 /(1+\mathrm{Gx} 2)$ and Supersymmetry, Phys. Lett. A, 122 (1987), 275-279.

[17] L.I. Schiff, "Quantum Mechanics", Mc Graw-Hill, New York, 1968.

[18] A. Sinha, R. Roychoudhury e Y.P. Varshni, Shifted 1/N expansion for confined quantum systems, Can. J. Phys., 78 (2000), 141-152.

[19] A. Sinha, R. Roychoudhury e Y.P. Varshni, WKB quantization rules for threedimensional confinement, Can. J. Phys., 79 (2001), 939-946.

[20] C.V. Sukumar, Supersymmetric Quantum-Mechanics of One-Dimensional Systems, J. Phys. A: Math. Gen., 18 (1985), 2917-2936.

[21] C.V. Sukumar, Supersymmetry, Factorization of the Schrödinger-Equation and a Hamiltonian Hierarchy, J. Phys. A: Math. Gen., 18 (1985), L57-L61.

[22] Y.P. Varshni, Accurate wavefunctions for hydrogenic donors in GaAs(Ga,Al)As quantum dots, Phys. Lett. A, 252 (1999), 248-250.

[23] Y.P. Varshni, Accurate wavefunctions for the confined hydrogen atom at high pressures, J. Phys. B: At. Mol. Opt. Phys., 30 (1997), L589-L593. 